Rigorous analysis of the Tomonaga model by means of Ward identities and the renormalization group

This article has been downloaded from IOPscience. Please scroll down to see the full text article.

J. Stat. Mech. (2005) L04001

(http://iopscience.iop.org/1742-5468/2005/04/L04001)

View the table of contents for this issue, or go to the journal homepage for more

Download details:

IP Address: 160.80.10.107

The article was downloaded on 16/06/2011 at 12:06

Please note that terms and conditions apply. 


\title{
LETTER
}

\section{Rigorous analysis of the Tomonaga model by means of Ward identities and the renormalization group}

\author{
Giuseppe Benfatto and Vieri Mastropietro \\ Dipartimento di Matematica, Università di Roma 'Tor Vergata', Viale della \\ Ricerca Scientifica 00133 Roma, Italy \\ E-mail: benfatto@axp.mat.uniroma2.it and mastropi@axp.mat.uniroma2.it
}

Received 4 March 2005

Accepted 6 April 2005

Published 12 April 2005

Online at stacks.iop.org/JSTAT/2005/L04001

doi:10.1088/1742-5468/2005/04/L04001

\begin{abstract}
The Tomonaga model describes interacting 1D fermions with a linear dispersion relation and a bandwidth cut-off, which destroys local gauge invariance and makes the model not solvable. We rigorously obtain its Schwinger functions by combining renormalization group analysis with Ward identities and a set of 'correction identities', which relate the correction terms to formal Ward identities (due to cut-offs) with some Schwinger functions. Contrary to previous cases, the use of the Luttinger model exact solution is completely avoided. Therefore this should be considered the first proof of what has been so far a conjecture: 1D interacting fermions can be constructed on the basis of a non-perturbative analysis independent of any exact solutions (of models which could be shown to have essentially the same beta function) and entirely based on a functional integral approach. The same method allows us to construct essentially all 1D Luttinger liquid models.
\end{abstract}

Keywords: Luttinger liquids (theory), spin chains, ladders and planes (theory) 


\section{Contents}

1. Introduction 2

2. Grassmann formulation of the Tomonaga model 3

3. Renormalization group analysis 5

4. Dyson and Ward identities $\quad 6$

$\begin{array}{ll}\text { References } & 9\end{array}$

\section{Introduction}

A well known problem in quantum field theory (QFT) is the basic conflict between regularizations and Ward identities (WI) based on local gauge symmetries. Dimensional regularization [11] apparently solves such a problem, but it seems inadequate for producing non-perturbative ${ }^{1}$ results. In a (Wilsonian) renormalization group (RG) approach, which is the best suited for getting non-perturbative results, such a conflict appears quite naturally; the functional integration is done by integrating fields of decreasing momentum scale, and hence one has to introduce suitable momentum cut-offs, which destroy the local gauge invariance necessary for deriving WI at any stage of the RG approach. On the other hand, if one does not use WI, the RG approach can be effective only for constructing trivial theories. QFT methods are used also in statistical physics; in such a case the problem is even more dramatic, as local gauge symmetry is broken even in the original model by natural cut-offs like a crystalline lattice or nonlinear bands.

The aim of this letter is to present the first example of rigorous implementation of WI based on local gauge invariance in a RG scheme. In particular we will obtain a general proof of Luttinger liquid behaviour (in the sense of [7] or [1]) for essentially all models of interacting spinless fermions for $d=1$. Of course the WI that we find at any stage of the RG approach are different to the formal ones, because of the presence of correction terms due to the cut-offs; such corrections apparently rob WI of their utility, which is their ability to give a non-trivial relation between different Schwinger functions. However, the new ingredient is a set of correction identities, relating the corrections to the formal WI with some Schwinger functions (up to smaller terms); this allows us to 'close the equations' and to reduce the number of independent running coupling constants. Detailed proofs of our statements are quite technical and will be published elsewhere [3]; we explain in this paper the main ideas. We will consider here for simplicity the Tomonaga model [10], which is the simplest non-solvable interacting Fermi system for $d=1$; it describes two kinds of (spinless) fermions (represented by two anticommuting operators $a_{k, \omega}^{ \pm}, \omega= \pm$ ) interacting with a local potential; they have a linear dispersion relation and their momenta $k$ are restricted to two regions around the two Fermi points by a bandwidth cut-off. The linear dispersion relation is simply a 'linearization' of a realistic

\footnotetext{
${ }^{1}$ By non-perturbative we mean that convergence of the power series can be proved.
} 
dispersion relation close to the two Fermi points, and the cut-off is imposed in order to avoid 'spurious' ultraviolet divergences. The reason is the presence of the bandwidth cutoff, which destroys many symmetries (conformal or local gauge invariance) which lie at the basis of many 'exact' methods; this is the same kind of difficulty one finds in generic more realistic Luttinger liquids. First of all, no solution using the Bethe ansatz has been found. In addition, the powerful techniques of bosonization cannot be applied; the reason is that the operators $\rho_{\omega}(p)=(1 / L) \sum_{k} a_{k+p, \omega}^{+} a_{k, \omega}^{-}$do not verify bosonic commutation relations, that is $\left[\rho_{\omega}(p), \rho_{\omega^{\prime}}\left(p^{\prime}\right)\right] \neq(p L / 2 \pi) \delta_{\omega, \omega^{\prime}} \delta_{p, p^{\prime}}$. Note that, if the bandwidth cut-off is removed and a smooth short range interaction is considered, one gets a famous variant of the Tomonaga model, the Luttinger model. After filling the 'Dirac sea' of unphysical states with negative energy, the operators $\rho_{\omega}(p)$ verify the exact commutation relation $\left[\rho_{\omega}(p), \rho_{\omega^{\prime}}\left(p^{\prime}\right)\right]=(p L / 2 \pi) \delta_{\omega, \omega^{\prime}} \delta_{p, p^{\prime}}$, and this immediately implies that the model can be solved [8]. As the bosonization method cannot really be applied to the Tomonaga model, its analysis was approached by other methods. Since a naive perturbation expansion is plagued by logarithmic divergences, perturbative RG methods [9] have been used to resum the perturbative series. However the flow of the running coupling constants is bounded only as a consequence of cancellations in the beta function, which were only checked at the third order; hence the results are, all the same, essentially perturbative. In $[2,4]$ several 1D Luttinger liquids were rigorously constructed, including the Tomonaga model; the observables are expressed as a convergent expansion in the running coupling constants, which remain in the domain of convergence as a consequence of a remarkable property, the vanishing of the beta function, deduced from the exact solution of the Luttinger model. However a major question remained open: is it possible to construct 1D Luttinger liquids without any use of exact solutions, only combining renormalization group methods and Ward identities? This question is quite relevant, as exact solutions are peculiar to 1D while the RG approach combined with WI is a general method which can be applied in principle in any dimension. Of course this goal is much more ambitious; instead of using the RG approach to prove that generic 1D interacting fermions are 'close' to the Luttinger model and then using non-trivial properties deduced from the exact solution, we want to show that, only employing the RG approach and WI, one can construct 1D non-solvable fermion models. In the physical literature it is claimed - see for instance $[5,6]$ - that this is really possible, by getting a set of closed equations combining Dyson equations and WI; however such analysis is not rigorous and, in any case, can be applied only to models without cut-offs. We show in this paper that such 'closure' is really possible, even in the presence of cut-offs, so obtaining the first example of complete construction of a non-trivial theory just by implementing WI in a RG approach.

\section{Grassmann formulation of the Tomonaga model}

The generating functional of the Schwinger functions of the Tomonaga model is given by the following Grassmann integral:

$\mathcal{W}(\phi, J)=\log \int P(\mathrm{~d} \psi) \exp \left(-V(\psi)+\sum_{\omega} \int \mathrm{d} \mathbf{x}\left[J_{\mathbf{x}, \omega} \psi_{\mathbf{x}, \omega}^{+} \psi_{\mathbf{x}, \omega}^{-}+\sum_{\varepsilon} \phi_{\mathbf{x}, \omega}^{+\varepsilon} \psi_{\mathbf{x}, \omega}^{-\varepsilon}\right]\right)$ 
Rigorous analysis of the Tomonaga model by means of Ward identities and the renormalization group

where $J_{\mathbf{x}, \omega}, \phi_{\mathbf{x}, \omega}^{ \pm}$are external fields, $\psi_{\mathbf{k}, \omega}^{ \pm}, \omega= \pm$ are Grassmann variables, $P(\mathrm{~d} \psi)$ is a fermionic Gaussian integration with propagator

$$
g_{\omega}(\mathbf{x}-\mathbf{y})=\int \mathrm{d} \mathbf{k} \mathrm{e}^{\mathrm{i} \mathbf{k}(\mathbf{x}-\mathbf{y})} \frac{\chi(\mathbf{k})}{-\mathrm{i} k_{0}+\omega k}
$$

where $\mathbf{x}=\left(x_{0}, x\right), \mathbf{k}=\left(k_{0}, k\right), \int \mathrm{d} \mathbf{k}=\frac{1}{\beta L} \sum_{\mathbf{k}}, \chi(\mathbf{k})$ a cut-off function and

$$
V(\psi)=\lambda \int \mathrm{d} \mathbf{x} \psi_{\mathbf{x},+}^{+} \psi_{\mathbf{x},+}^{-} \psi_{\mathbf{x},-}^{+} \psi_{\mathbf{x},-}^{-} .
$$

The cut-off $\chi(\mathbf{k})$ is chosen in the following way; if $\chi_{0}(t)$ is a smooth compact support function equal to 1 for $0<t<1$ and vanishing for $t \geq \gamma>1$, we define $f_{i}(\mathbf{k})=$ $\chi_{0}\left(\gamma^{-i}|\mathbf{k}|\right)-\chi_{0}\left(\gamma^{-i+1}|\mathbf{k}|\right)$ (with support in $\left.\gamma^{i-1} \leq|\mathbf{k}| \leq \gamma^{i+1}\right)$ and $\chi(\mathbf{k})=\sum_{i=h}^{0} f_{i}(\mathbf{k})$; then $\chi(\mathbf{k})$ has support between $\gamma^{h-1}$ (the infrared (IR) cut-off, $h \leq 0$ ) and $\gamma$ (the ultraviolet $(U V)$ cut-off). The Schwinger functions of the Tomonaga model are found by removing the IR cut-off $(h \rightarrow-\infty)$ while keeping the UV cut-off fixed. Note that we have also imposed an energy cut-off, in addition to the bandwidth cut-off; this is done only for notational convenience and can be easily avoided. The Schwinger functions relevant in the subsequent discussion are defined in the following way:

$$
\begin{aligned}
& G_{\omega}^{2,1}(\mathbf{x} ; \mathbf{y}, \mathbf{z})=\left.\frac{\partial}{\partial J_{\mathbf{x}, \omega}} \frac{\partial^{2}}{\partial \phi_{\mathbf{y},+}^{+} \partial \phi_{\mathbf{z},+}^{-}} \mathcal{W}(\phi, J)\right|_{J=0, \phi=0} \\
& G_{\omega}^{4}\left(\mathbf{x}_{1}, \mathbf{x}_{2}, \mathbf{x}_{3}, \mathbf{x}_{4}\right)=\left.\frac{\partial^{4} \mathcal{W}}{\partial \phi_{\mathbf{x}_{1}, \omega}^{+} \partial \phi_{\mathbf{x}_{2}, \omega}^{-} \partial \phi_{\mathbf{x}_{3},-\omega}^{+} \partial \phi_{\mathbf{x}_{4},-\omega}^{-}}\right|_{J=0, \phi=0} \\
& G_{\omega}^{2}(\mathbf{y}, \mathbf{z})=\left.\frac{\partial^{2}}{\partial \phi_{\mathbf{y}, \omega}^{+} \partial \phi_{\mathbf{z}, \omega}^{-}} \mathcal{W}(\phi, J)\right|_{J=0, \phi=0} .
\end{aligned}
$$

By Taylor expanding $\mathrm{e}^{-V}$, one sees that the Schwinger functions are expressed as power series in $\lambda$; such series are indeed convergent, as a consequence of the Gram-Hadamard bound for fermionic expectations, but a naive bound only allows one to get a convergence radius which shrinks to 0 as $h \rightarrow-\infty$, that is when the IR cut-off is removed. The problem is to show that it is possible to rewrite the Schwinger functions in terms of suitable effective couplings, so that the new expansions (which are not series expansions) can be bounded uniformly in the infrared cut-off.

From equation (1) one can immediately derive Ward identities from the chiral gauge transformation $\psi_{\mathbf{x},-}^{ \pm} \rightarrow \psi_{\mathbf{x},-}^{ \pm}, \psi_{\mathbf{x},+}^{ \pm} \rightarrow \mathrm{e}^{ \pm \alpha_{\mathbf{x}}} \psi_{\mathbf{x},+}^{ \pm}$; one gets, see figure 1,

$$
D_{+}(\mathbf{p}) \hat{G}_{+}^{2,1}(\mathbf{p}, \mathbf{k}, \mathbf{q})=\hat{G}_{+}^{2}(\mathbf{q})-\hat{G}_{+}^{2}(\mathbf{k})+\hat{\Delta}_{+}^{2,1}(\mathbf{p}, \mathbf{k}, \mathbf{q})
$$

where $\Delta_{+}^{2,1}(\mathbf{p}, \mathbf{k}, \mathbf{q})$ is given by

$$
\int \mathrm{d} \mathbf{k} C_{+}(\mathbf{k}, \mathbf{k}-\mathbf{p})\left\langle\hat{\psi}_{\mathbf{k},+}^{+} \hat{\psi}_{\mathbf{k}-\mathbf{p},+}^{-} ; \hat{\psi}_{\mathbf{k},+}^{-} \hat{\psi}_{\mathbf{q},+}^{+}\right\rangle^{\mathrm{T}}
$$

with $C_{\omega}(\mathbf{k}, \mathbf{q})=\left[\chi^{-1}(\mathbf{k})-1\right] D_{\omega}(\mathbf{k})-\left[\chi^{-1}(\mathbf{q})-1\right] D_{\omega}(\mathbf{q})$ and $D_{\omega}(\mathbf{k})=-\mathrm{i} k_{0}+\omega k$. Note that the above WI differs from the formal one for the presence of the correction term $\Delta_{+}^{2,1}(\mathbf{p}, \mathbf{k}, \mathbf{q})$; such a term is not negligible, as its series expansion is logarithmically diverging as was the series for the Schwinger functions itself. The correction term $\hat{\Delta}_{+}^{2,1}$ is non-vanishing even if both UV and IR cut-offs are removed; this is not too surprising as it is well known that WI based on chiral gauge transformations in QFT are plagued by anomalies. 


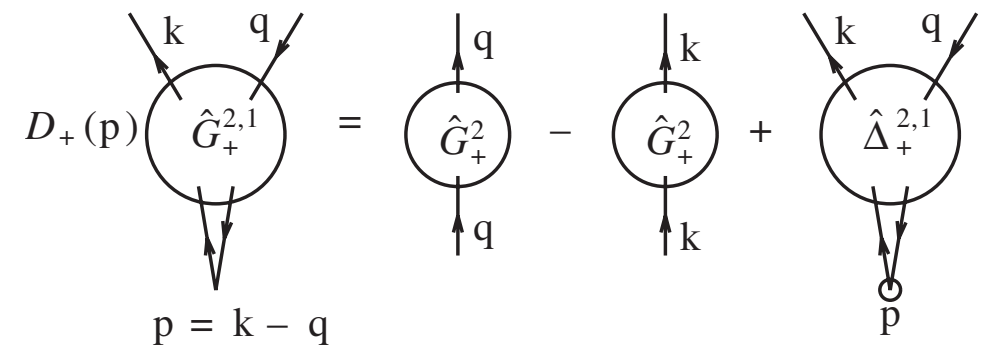

Figure 1. The Ward identity (5); the small circle represents $C_{+}$.

\section{Renormalization group analysis}

We evaluate the Grassmann integral equation (1) by an exact $R G$ procedure, described in detail elsewhere [4]. The Grassmann integration is expressed as the product of many independent integrations, by using the addition property for Grassmann integrals $P(\mathrm{~d} \psi)=\prod_{i=h}^{0} P\left(\mathrm{~d} \psi^{(i)}\right)$, with $P\left(\mathrm{~d} \psi^{(i)}\right)$ a fermionic integration with propagator $g_{\omega}^{(i)}(\mathbf{k})=$ $f^{(i)}(\mathbf{k}) / D_{\omega}(\mathbf{k})$. Let us consider first for simplicity the integration of the partition function, given by the $\log$ argument in equation (1), with $J=\phi=0$; after the fields $\psi^{(0)}, \psi^{(-1)}, \ldots, \psi^{(i)}, \mathrm{i}>h$ are integrated we get

$$
\int P_{Z_{i}}\left(\psi^{(\leq i)}\right) \mathrm{e}^{-V^{(i)}\left(\sqrt{Z_{i}} \psi^{(\leq i)}\right)}=\int P_{Z_{i}}\left(\psi^{(\leq i)}\right) \mathrm{e}^{-\mathcal{L} V^{(i)}\left(\sqrt{Z_{i}} \psi^{(\leq i)}\right)-\mathcal{R} V^{(i)}\left(\sqrt{Z_{i}} \psi^{\leq i}\right)}
$$

where $\int P_{Z_{i}}(\psi \leq i)$ is the Grassmann integration with propagator

$$
\frac{1}{Z_{i}} g_{\omega}^{(\leq i)}(\mathbf{x}-\mathbf{y})=\frac{1}{Z_{i}} \sum_{j=h}^{i} \int \mathrm{d} \mathbf{k} \mathrm{e}^{\mathrm{i} \mathbf{k}(\mathbf{x}-\mathbf{y})} \frac{f^{(j)}(\mathbf{k})}{-\mathrm{i} k_{0}+\omega k} .
$$

Moreover $V^{(i)}$, the effective potential at scale $i$, is given by a sum of integrals of monomials of any order in the $\psi_{\mathbf{k}, \omega}$ variables, times suitable kernels (functions of the fermionic momenta); $\mathcal{L}$ is the localization operator, whose action is to replace the kernels multiplying quartic or quadratic monomials with their zeroth- or first-order Taylor expansion around $(0,0)$, respectively; finally $\mathcal{R}=1-\mathcal{L}$. Then we get

$\mathcal{L} \mathcal{V}^{(i)}(\psi)=l_{i} \int \mathrm{d} \mathbf{x} \psi_{+, \mathbf{x}}^{+} \psi_{+, \mathbf{x}}^{-} \psi_{-, \mathbf{x}}^{+} \psi_{-, \mathbf{x}}^{-}+z_{i} \sum_{\omega= \pm 1} \int \mathrm{d} \mathbf{x} \psi_{\omega, \mathbf{x}}^{+}\left(\partial_{x_{0}}+\mathrm{i} \omega \partial_{x}\right) \psi_{\omega, \mathbf{x}}^{-}$

and both terms are dimensionally marginal in the RG sense. Calling the first addend in equation $(9) \lambda_{i} F_{\lambda}(\psi)$ and moving the quadratic part from the interaction to the integration in equation (7) we get an expression equivalent to the following one (see [4] for a precise definition):

$$
\begin{aligned}
\left.\int P_{Z_{i-1}}\left(\psi^{(\leq i)}\right) \mathrm{e}^{-l_{i} F_{\lambda}\left(\sqrt{Z_{i}} \psi^{\leq i}\right)-\mathcal{R} V^{(i)}\left(\sqrt{Z_{i}} \psi \leq i\right.}\right) \\
\\
\left.\equiv \int P_{Z_{i-1}}\left(\psi^{(\leq i-1)}\right) \int P_{Z_{i-1}}\left(\psi^{(i)}\right) \mathrm{e}^{-\lambda_{i} F_{\lambda}\left(\sqrt{Z_{i-1}} \psi^{\leq i}\right)-\mathcal{R} V^{(i)}\left(\sqrt{Z_{i}} \psi \leq i\right.}\right)
\end{aligned}
$$

where $Z_{i-1}=Z_{i}\left(1+z_{i}\right)$ and $\lambda_{i}=\left(Z_{i} / Z_{i-1}\right)^{2} l_{i}$. The integration over $\psi^{(i)}$, with propagator $Z_{i-1}^{-1} g_{\omega}^{(i)}(\mathbf{x}-\mathbf{y})$, can be done using the invariance of exponentials property and we get that 
the last integral in the rhs of equation (10) has the form $\mathrm{e}^{-\mathcal{V}^{(i-1)}\left(\psi^{(\leq i-1)}\right)}$; the procedure can be then iterated. The integration of the generating functional $\mathcal{W}$ of equation (1) is done in a similar way; in the effective potential there appear terms depending also on $\phi$ or $J$, and $\mathcal{L}$ acts non-trivially on the terms bilinear in $\psi$ and linear in $J$; the resulting local terms are of the form $Z_{i}^{(2)} J \psi_{\omega}^{+} \psi_{\omega}^{-}$, the coupling $Z_{i}^{(2)}$ being the density renormalization. A crucial result, proved in [4] using the Gram-Hadamard bound for determinants applied to the fermionic truncated expectations, is the following:

The Schwinger functions are expressed as expansions in the running coupling constants (rcc) $\left\{\lambda_{j}\right\}_{j=0,-1, \ldots, h}$, which are convergent uniformly in the IR cut-off, if $\sup _{j \geq h}\left|\lambda_{j}\right|$ is small enough.

The $\mathrm{RG}$ integration procedure gives a resummation of the naive series expansion; instead of a power series in $\lambda$ (whose convergence radius is apparently vanishing as $h \rightarrow-\infty)$, we have found a new expansion in terms of $\left\{\lambda_{j}\right\}_{j=0,-1, \ldots, h}$, which is not a power expansion but is convergent uniformly in the IR cut-off. Such an expansion implies that the Schwinger functions are indeed analytic in the original coupling (with a radius of convergence depending on the external momenta and going to zero at the singular points) and provides very detailed information on their asymptotic behaviour. For instance we get, in the limit $h=-\infty$ and assuming that $\sup _{j}\left|\lambda_{j}\right| \leq C|\lambda|$, for $|\mathbf{x}-\mathbf{y}| \geq 1$

$$
G_{\omega}^{2}(\mathbf{x}, \mathbf{y})=\frac{g_{\omega}(\mathbf{x}-\mathbf{y})}{|\mathbf{x}-\mathbf{y}|^{\eta}}(1+\lambda A(\mathbf{x}, \mathbf{y}))
$$

with $\eta=a \lambda^{2}+\mathrm{O}\left(\lambda^{3}\right), a>0$ and $|A(\mathbf{x}, \mathbf{y})| \leq C$. The main problem at this stage is then: what ensures that $\left\{\lambda_{j}\right\}_{j=0,-1, \ldots, h}$ are bounded and $\mathrm{O}(\lambda)$ uniformly in the IR cut-off $h$ ?

\section{Dyson and Ward identities}

The property that the $\operatorname{rcc} \lambda_{j}$ remain small for any $j$ is not trivial at all. The rec verify a recursive equation whose rhs is called a beta function; one can verify by an explicit computation that, up to two loops, dramatic cancellations are present; however, if at higher orders the cancellations were not present, the rcc could increase without limit making the analysis meaningless. One needs then a non-perturbative argument. First note that the $\operatorname{rcc} \lambda_{j}$ for $j>h$ are identical in the model with or without an IR cutoff; this is a simple consequence of our compact support decomposition. Then finding a relation between the Schwinger functions at momenta computed at the cut-off scale $\gamma^{h}$ in the model with IR cut-off $\gamma^{h-1}$, we get a relation between the rcc at scale $h$ for the model without a cut-off scale. In a sense, we are using the idea of the multiplicative RG in the context of the Wilsonian RG.

As $\hat{G}^{4}$, computed at momenta at the cut-off scale, is proportional to $\lambda_{h}$, that is, see [3], $\hat{G}^{4}\left(\overline{\mathbf{k}}_{1}, \overline{\mathbf{k}}_{2}, \overline{\mathbf{k}}_{3}, \overline{\mathbf{k}}_{4}\right)=\left(\lambda_{h} / Z_{h}^{2}\right) \gamma^{-4 h}\left(1+\mathrm{O}\left(\bar{\lambda}_{h}\right)\right)$, where $\left|\overline{\mathbf{k}}_{i}\right|=\gamma^{h}$ and $\bar{\lambda}_{h}=\sup _{j \geq h}\left|\lambda_{j}\right|$, it is natural to write a Dyson equation for $G^{4}$, see figure 2 :

$$
\begin{gathered}
-\hat{G}_{+}^{4}\left(\mathbf{k}_{1}, \mathbf{k}_{2}, \mathbf{k}_{3}, \mathbf{k}_{4}\right)=\lambda \hat{g}_{-}\left(\mathbf{k}_{4}\right)\left[\hat{G}_{-}^{2}\left(\mathbf{k}_{3}\right) \hat{G}_{+}^{2,1}\left(\mathbf{k}_{1}-\mathbf{k}_{2}, \mathbf{k}_{1}, \mathbf{k}_{2}\right)\right. \\
\left.+\int \mathrm{d} \mathbf{p} \hat{G}_{+}^{4,1}\left(\mathbf{p} ; \mathbf{k}_{1}, \mathbf{k}_{2}, \mathbf{k}_{3}, \mathbf{k}_{4}-\mathbf{p}\right)\right]
\end{gathered}
$$




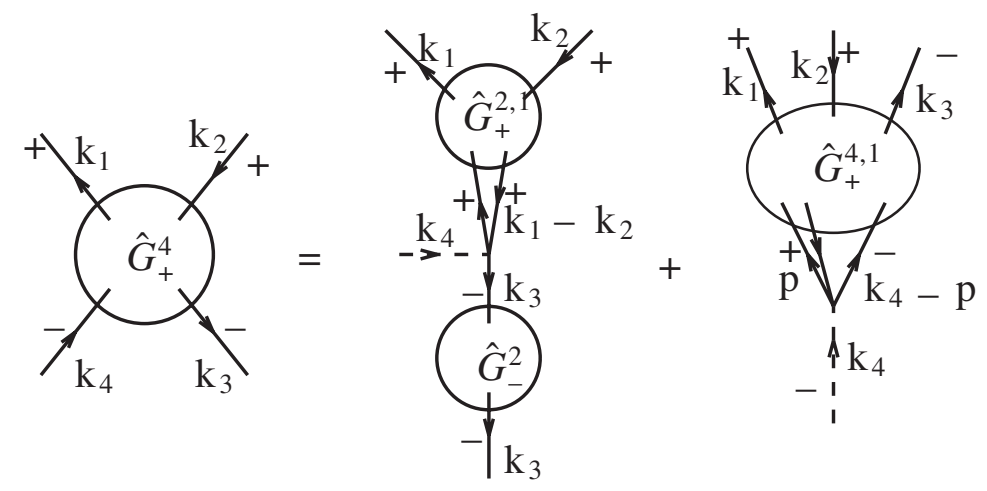

Figure 2. Graphical representation of the Dyson equation.

The lhs of equation (12) is proportional to $\lambda_{h}$, while the rhs is proportional to $\lambda$; however, if we do not take into account cancellations, we get from equation (12) simply $\left|\lambda_{h}\right| \leq C_{h}|\lambda|$, with $C_{h} \rightarrow \infty$ as $h \rightarrow-\infty$. We can however express $\hat{G}_{+}^{2,1}$ and $\hat{G}_{+}^{4,1}$ in terms of $\hat{G}_{+}^{2}$ and $\hat{G}_{+}^{4}$ by means of WI (5) and

$$
\begin{gathered}
D_{+}(\mathbf{p}) G_{+}^{4,1}\left(\mathbf{p}, \mathbf{k}_{1}, \mathbf{k}_{2}, \mathbf{k}_{3}, \mathbf{k}_{4}-\mathbf{p}\right)=G_{+}^{4}\left(\mathbf{k}_{1}-\mathbf{p}, \mathbf{k}_{2}, \mathbf{k}_{3}, \mathbf{k}_{4}-\mathbf{p}\right) \\
-G_{+}^{4}\left(\mathbf{k}_{1}, \mathbf{k}_{2}+\mathbf{p}, \mathbf{k}_{3}, \mathbf{k}_{4}-\mathbf{p}\right)+\Delta_{+}^{4,1} .
\end{gathered}
$$

If the formal WI were true (that is if $\hat{\Delta}_{+}^{2,1}$ and $\hat{\Delta}_{+}^{4,1}$ were vanishing), by inserting the formal WI in (12) one would get at once, just by dimensional analysis, $\lambda_{h}=\lambda+\mathrm{O}\left(\lambda^{2}\right)$. However $\hat{\Delta}_{+}^{2,1}$ and $\hat{\Delta}_{+}^{4,1}$ are not negligible at all; their naive power series expansion is even log diverging and this seems to completely rob the WI of their utility (one cannot 'close the equations'). Luckily there are other remarkable relations, which we call correction identities, connecting the correction term $\hat{\Delta}_{+}^{2,1}, \hat{\Delta}_{+}^{4,1}$ to the Schwinger functions; for instance $\hat{\Delta}_{+}^{2,1}$ can be written as, see figure 3 ,

$$
\hat{\Delta}_{+}^{2,1}(\mathbf{p}, \mathbf{k}, \mathbf{q})=\nu_{+} D_{+}(\mathbf{p}) \hat{G}_{+}^{2,1}+\nu_{-} D_{-}(\mathbf{p}) \hat{G}_{-}^{2,1}+\hat{H}_{+}^{2,1}(\mathbf{p}, \mathbf{k}, \mathbf{q})
$$

where $\nu_{+}, \nu_{-}$are $\mathrm{O}(\lambda)$, uniformly in $h$, and $\hat{H}_{+}^{2,1}(\mathbf{p}, \mathbf{k}, \mathbf{q})$ can be obtained from a functional integral $\tilde{\mathcal{W}}$ very similar to $(1)$, with the difference that the term proportional to $J$ in the exponent is replaced by

$$
\int \mathrm{d} \mathbf{p} \mathrm{d} \mathbf{k} C_{+}(\mathbf{k}, \mathbf{k}-\mathbf{p}) \hat{J}_{\mathbf{p}} \psi_{\mathbf{k},+}^{+} \psi_{\mathbf{k}-\mathbf{p},+}^{+}-\sum_{\omega} \nu_{\omega} \int \mathrm{d} \mathbf{p} \mathrm{d} \mathbf{k} \hat{J}_{\mathbf{p}} \psi_{\mathbf{k}, \omega}^{+} \psi_{\mathbf{k}-\mathbf{p}, \omega}^{+} .
$$

The multiscale integration of $\tilde{\mathcal{W}}$ (such that $\left.H_{+}^{2,1}=\left.\left(\partial / \partial J_{\mathbf{x}, \omega}\right)\left(\partial^{2} / \partial \phi_{\mathbf{y},+}^{+} \partial \phi_{\mathbf{z},+}^{-}\right) \tilde{\mathcal{W}}(\phi, J)\right|_{0,0}\right)$ is done in a similar way to the one used for (1) in section 3, described in detail in [3] and based on the peculiar properties of $C_{+}\left(\mathbf{k}_{+}, \mathbf{k}_{-}\right)$, in particular that $C_{+}\left(\mathbf{k}_{+}, \mathbf{k}_{-}\right) g^{(i)}\left(\mathbf{k}_{+}\right) g^{(j)}\left(\mathbf{k}_{-}\right)$is vanishing unless $i$ or $j$ are equal to 0 or $h$ (that is, the infrared or ultraviolet cut-off scale). New marginal terms $\nu_{j, \omega} \int \mathrm{d} \mathbf{p} \mathrm{d} \mathbf{k} \hat{J}_{\mathbf{p}} \psi_{\mathbf{k}, \omega}^{(\leq j)+} \psi_{\mathbf{k}-\mathbf{p}, \omega}^{(\leq j)-}$, $\omega= \pm$, are generated in the integration procedure, and the $\nu_{\omega}$ are fixed so that $\nu_{j, \omega}=\mathrm{O}\left(\lambda \gamma^{\vartheta j}\right)$ with $0<\vartheta<1$. At the end the bound $\left|\hat{H}_{+}^{2,1}(\overline{\mathbf{p}}, \overline{\mathbf{k}}, \overline{\mathbf{q}})\right| \leq C \bar{\lambda}_{h}\left(\gamma^{2 h} / Z_{h}\right)$ is found. The physical meaning of the first two terms or the third one in the correction 
Rigorous analysis of the Tomonaga model by means of Ward identities and the renormalization group

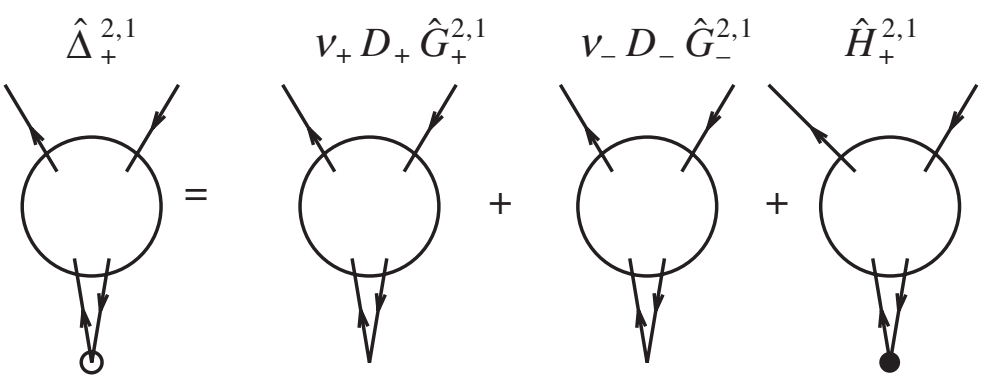

Figure 3. Graphical representation of the correction identity; the filled point represents equation (15).
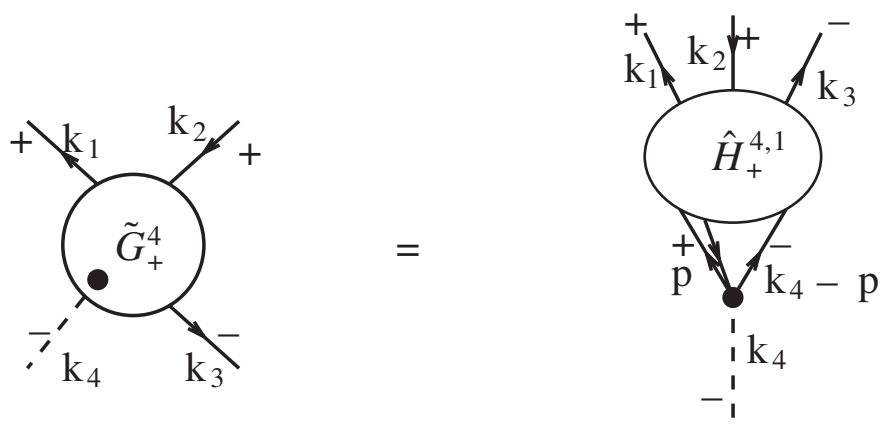

Figure 4. The Dyson equation (19); the filled point represents $T_{1}+\sum_{\omega} T_{\omega}$.

identity equation (14) is completely different. The first two terms are related to the chiral anomaly, which produces a correction to the formal WI even if $\chi=1$ and the model reduces to a QFT model; in fact, if the cut-offs are removed, $\nu_{ \pm}$tends to a non-vanishing limit. On the other hand, the third term takes into account the presence of finite cut-offs, and it is vanishing on removing them at fixed external momenta. If we insert the correction identity (14) in the WI (5), we find $\left(1-\nu_{+}\right) D_{+}(\mathbf{p}) \hat{G}_{+}^{2,1}-\nu_{-} D_{-}(\mathbf{p}) \hat{G}_{-}^{2,1}=\hat{G}_{+}^{2}(\mathbf{q})-\hat{G}_{+}^{2}(\mathbf{k})+$ $\hat{H}_{+}^{2,1}$. A WI holds also for $G_{-}^{2,1}$, that is $\left(1-\nu_{-}^{\prime}\right) D_{-}(\mathbf{p}) G_{-}^{2,1}-\nu_{+}^{\prime} D_{-}(\mathbf{p}) G_{+}^{2,1}=H_{-}^{2,1}$, and by using that $G^{2}(\overline{\mathbf{k}})=\gamma^{-h} Z_{h}^{-1}\left(1+\mathrm{O}\left(\bar{\lambda}_{h}\right)\right)$ and that $\left|\hat{H}_{ \pm}^{2,1}\right| \leq C \bar{\lambda}_{h} Z_{h}^{-1} \gamma^{-2 h}$, we get that the first addend of the rhs of the Dyson equation is given by $\lambda \gamma^{-4 h} Z_{h}^{-2}\left(1+\mathrm{O}\left(\bar{\lambda}_{h}\right)\right)$, which is the 'good bound' one needs.

The analysis of the second addend of the rhs of (12) is more complex. Also the correction $\hat{\Delta}_{+}^{4,1}$ to the WI equation (16) verifies a correction identity, as we proved in [4]:

$$
\hat{\Delta}_{+}^{4,1}=\nu_{+} D_{+}(\mathbf{p}) \hat{G}_{+}^{4,1}+\nu_{-} D_{-}(\mathbf{p}) \hat{G}_{-}^{4,1}+\hat{H}_{+}^{4,1} .
$$

One has then to combine the WI (13), the one for $\hat{G}_{-}^{4,1}$ and the correction identities (16) and $\hat{\Delta}_{-}^{4,1}=\nu_{+}^{\prime} D_{+}(\mathbf{p}) \hat{G}_{+}^{4,1}+\nu_{-}^{\prime} D_{-}(\mathbf{p}) \hat{G}_{-}^{4,1}+\hat{H}_{-}^{4,1}$. After some algebra one gets

$$
\begin{aligned}
& \frac{D_{+}(\mathbf{p}) \hat{G}_{+}^{4,1}}{1+A}\left(\mathbf{p}, \mathbf{k}_{1}, \mathbf{k}_{2}, \mathbf{k}_{3}, \mathbf{k}_{4}-\mathbf{p}\right)=\hat{H}^{4,1}+\hat{G}_{+}^{4}\left(\mathbf{k}_{1}-\mathbf{p}, \mathbf{k}_{2}, \mathbf{k}_{3}, \mathbf{k}_{4}-\mathbf{p}\right) \\
& -\hat{G}_{+}^{4}\left(\mathbf{k}_{1}, \mathbf{k}_{2}+\mathbf{p}, \mathbf{k}_{3}, \mathbf{k}_{4}-\mathbf{p}\right)+B\left[\hat{G}_{+}^{4}\left(\mathbf{k}_{1}, \mathbf{k}_{3}-\mathbf{p}, \mathbf{k}_{4}-\mathbf{p}\right)\right. \\
& \left.-\hat{G}_{+}^{4}\left(\mathbf{k}_{1}, \mathbf{k}_{2}, \mathbf{k}_{3}, \mathbf{k}_{4}\right)\right]
\end{aligned}
$$


Rigorous analysis of the Tomonaga model by means of Ward identities and the renormalization group where $\hat{H}^{4,1}=\hat{H}_{+}^{4,1}+B \hat{H}_{-}^{4,1}, A=-\nu_{+}-\left(\nu_{-} \nu_{+}^{\prime}\right) /\left(1-\nu_{-}^{\prime}\right)$ and $B=\nu_{-} /\left(1-\nu_{-}^{\prime}\right)$. Inserting this expression in the second addend of the rhs of (12), we get several terms; the term involving $\hat{G}_{+}^{4}\left(\mathbf{k}_{1}, \mathbf{k}_{2}, \mathbf{k}_{3}, \mathbf{k}_{4}\right)$ is vanishing, while all the other terms involving $\hat{G}_{+}^{4}$ verify the dimensional bound $\left|\lambda g\left(\overline{\mathbf{k}}_{4}\right) \int \mathrm{d} \mathbf{p} \hat{G}_{+}^{4} D_{+}(\mathbf{p})^{-1}\right| \leq \bar{\lambda}_{h}^{2} Z_{h}^{-2} \gamma^{-4 h}$. The terms requiring a subtle analysis are the terms

$$
g\left(\mathbf{k}_{4}\right) \int \mathrm{d} \mathbf{p} \hat{H}_{ \pm}^{4,1} D_{+}(\mathbf{p})^{-1} .
$$

Their analysis is done by writing for them another Dyson equation (see figure 4):

$$
g_{-}\left(\mathbf{k}_{4}\right) \int \mathrm{d} \mathbf{p} \hat{H}_{+}^{4,1} D_{+}(\mathbf{p})^{-1}=\tilde{G}_{+}^{4}
$$

where $\tilde{G}_{+}^{4}=\left.\frac{\partial^{4}}{\partial \phi_{+}^{+} \partial \phi_{+}^{-} \partial \phi_{-}^{+} \partial J} \tilde{W}\right|_{0}$ and $\tilde{W}$ is similar to $W$ of equation (1), with $\int \mathrm{d} \mathbf{x} J \psi^{+} \psi^{-}$in the exponent replaced by $T_{1}+\sum_{\omega} \nu_{\omega} T_{\omega}$, where

$$
\begin{aligned}
T_{1} & =\int \mathrm{d} \mathbf{k} \mathrm{d} \mathbf{p} \frac{C_{+}(\mathbf{k}, \mathbf{k}-\mathbf{p})}{D_{+}(\mathbf{p})} \psi_{\mathbf{k},+}^{+} \psi_{\mathbf{k}-\mathbf{p},+}^{-} \psi_{\mathbf{k}_{4}-\mathbf{p},-}^{+} J_{\mathbf{k}_{4}} \hat{g}_{-}\left(\mathbf{k}_{4}\right) \mathbf{n} \\
T_{\omega} & =\int \mathrm{d} \mathbf{k} \mathrm{d} \mathbf{p} \frac{D_{\omega}(\mathbf{p})}{D_{+}(\mathbf{p})} \psi_{\mathbf{k}, \omega}^{+} \psi_{\mathbf{k}-\mathbf{p}, \omega}^{-} \psi_{\mathbf{k}_{4}-\mathbf{p},-}^{+} J_{\mathbf{k}_{4}} \hat{g}_{-}\left(\mathbf{k}_{4}\right) .
\end{aligned}
$$

The role of equation (19) is to relate the integral of $\hat{H}_{+}^{4,1}$ to a Grassmann integral similar to the one for $\hat{G}^{4}$ with a 'special' external line coming from the 'special' vertex associated with $T_{1}+\sum_{\omega} \nu_{\omega} T_{\omega}$. Also $\tilde{G}^{4}$ can be studied via a multiscale integration similar to the one in section 2; we refer the reader to [3] for the technical details. The presence of the special external line has the effect that new marginal terms appear, with which new running coupling constants must be associated; in particular a new running coupling constant multiplying a monomial trilinear in $\psi$ appears, which we call $\tilde{\lambda}_{i}$, and the analysis of its flow could be a problem; however, by using again the properties of the function $C_{\omega}$, it can be shown that it is essentially proportional to $\lambda_{i}$, that is $\left|\tilde{\lambda}_{i}-\alpha \lambda_{i}\right| \leq C \bar{\lambda}_{h} \gamma^{\vartheta i}$. Then one gets for $\tilde{G}_{4}$ a bound very similar to the one for $\hat{G}_{4}$, except that $\lambda_{h}$ is replaced by $\tilde{\lambda}_{h}$ and there is no wavefunction renormalization associated with the external line with momentum $\mathbf{k}_{4}$, i.e. there is a $Z_{h}^{-1}$ missing; we can however identify two classes of terms in the expansion of $\tilde{G}_{4}$, one in which the special external line comes out from a $\tilde{\lambda}_{i}$ vertex, and the other one such that it comes out from $\tilde{z}_{i}$, the local parts of the terms with two external lines (linear in $\psi$ ); by summing such terms one gets the bound $C \bar{\lambda}_{h}^{2} \gamma^{-4 h} Z_{h}^{-2}$, and this allows us to complete the proof that $\lambda_{h}=\lambda+\mathrm{O}\left(\lambda^{2}\right)$ for any $h$. Hence the expansions for the Schwinger functions are convergent, as explained in section 3, and equation (11) holds.

\section{References}

[1] Affleck I, 1984 Proc. Les Houches Summer School on Critical Phenomena, Random Systems, Gauge Theories (Amsterdam: North-Holland)

[2] Benfatto G, Gallavotti G, Procacci A and Scoppola B, 1993 Commun. Math. Phys. 16093

[3] Benfatto G and Mastropietro V, 2004 Preprint cond-mat/0409049

[4] Benfatto G and Mastropietro V, 2001 Rev. Math. Phys. 13 1323-435

Benfatto G and Mastropietro V, 2002 Commun. Math. Phys. 23197

Benfatto G and Mastropietro V, 2004 J. Stat. Phys. 115143 
Rigorous analysis of the Tomonaga model by means of Ward identities and the renormalization group

[5] Dzyaloshinky A E and Larkin A I, 1974 Sov. Phys.-JETP 38 202-8

[6] Di Castro C and Metzner W, 1991 Phys. Rev. Lett. 673852

[7] Haldane F D M, 1980 Phys. Rev. Lett. 451358

[8] Mattis D and Lieb E, 1965 J. Math. Phys. 6304

[9] Solyom J, 1979 Adv. Phys. 28201

[10] Tomonaga S I, 1950 Prog. Theor. Phys. 5544

[11] Zinn-Justin J, 1989 Quantum Field Theory and Critical Phenomena (Oxford: Oxford University Press) 